\title{
Self-similar vortex filament motion under the non-local Biot-Savart model
}

\author{
Robert A. Van Gorder $\dagger$ \\ Mathematical Institute, University of Oxford, Andrew Wiles Building, Radcliffe Observatory \\ Quarter, Woodstock Road, Oxford OX2 6GG United Kingdom \\ (Received ?; revised ?; accepted ?. - To be entered by editorial office)
}

One type of thin vortex filament structure that has attracted interest in recent years is that which obeys self-similar scaling. Among various applications, these filaments have been used to model the motion of quantized vortex filaments in superfluid Helium after reconnection events. While similarity solutions have been described analytically and numerically using the local induction approximation (LIA), they have not been studied (or even shown to exist) under the non-local Biot-Savart model. In this present paper, we show not only that self-similar vortex filament solutions exist for the non-local BiotSavart model, but that such solutions are qualitatively similar to their LIA counterparts. This suggests that the various LIA solutions found previously should be valid physically (at least in the small amplitude regime), since they agree well with the more accurate Biot-Savart model.

\section{Introduction}

The mathematical formulation for the self-induced motion of a thin vortex filament was given by Da Rios (1906) and rediscovered by others later (Arms \& Hama, 1965; Ricca, 1991). The dynamics of such a classical (Widnall, 1972) or a quantized vortex filament in the very low-temperature limit of superfluid Helium (Boffetta et al., 2009) vortex filament are governed by the Biot-Savart law

$$
\mathbf{r}_{t}=\frac{\kappa}{4 \pi} \int_{\ell} \frac{(\mathbf{s}-\mathbf{r}) \times d \mathbf{s}}{|\mathbf{s}-\mathbf{r}|^{3}}
$$

where curve $\mathbf{r}$ represents the spatial curve modeling the vortex filament at any time $t$, $\kappa$ denotes the strength of the filament (circulation), and $\ell$ is the path along which the filament lies. This representation is non-local, meaning that distant parts of the filament can effect the behavior of the filament at a specific point. The non-locality of this equation makes it difficult to solve for arbitrary vortex filament configurations, therefore the local induction approximation (LIA) is sometimes used to replace the integral in (1.1) with a far simpler relation, viz., the vector partial differential equation

$$
\mathbf{r}_{t}=\frac{\kappa}{4 \pi} \ln \left(1 / \delta^{2}\right) \mathbf{t} \times \mathbf{n},
$$

where $\mathbf{t}$ is the tangent vector, $\mathbf{n}$ is the normal vector to $\mathbf{r}$ scaled with the scalar curvature. Here $\delta$ is a small distance parameter signifying that we have removed the logarithmic singularity and are approximating the region near this singularity. The right hand side of (1.2) is the binormal vector (up to scaling). While (1.2) defines a nonlinear vector partial differential equation, its solution is still simpler than that of the non-local and singular equation (1.1). The LIA is useful when the vortex filaments are very thin, which is true

\footnotetext{
$\dagger$ Email address for correspondence: Robert.VanGorder@maths.ox.ac.uk
} 
for instance in the case of quantized vortex filaments in superfluid helium, provided that they are not too tightly coiled (Van Gorder, 2014), while for more tightly coiled filaments the Biot-Savart dynamics are needed (Van Gorder, 2015a). For classical vortex filaments, the LIA has well-known limitations, hence solution to the full Biot-Savart dynamics (1.1) are desirable where possible.

Due to the non-local and nonlinear nature of (1.1), few types of solutions are known. The most common solutions are closed vortex rings (Moin, Leonard, \& Kim, 1986; Adachi, Fujiyama, \& Tsubota, 2010) and open helical vortex filaments (Boersma \& Wood, 1999; Ricca, 1994; Van Gorder, 2015b; Widnall, 1972). Recently, it was shown that planar filaments are possible under the non-local Biot-Savart dynamics (Van Gorder, 2015c).

Self-similar solutions to the LIA have been studied in relation the the dynamics of quantized vortex filaments in superfluid Helium (Bewley et al., 2008; Lipniacki, 2000, 2003a,b; Van Gorder, 2013). Such solutions are interesting as they can model sharp kinks along vortex filaments, as well as the development of singularities (Gutiérrez, Rivas, \& Vega, 2003). Pelz (1997) numerically simulated vortex tangles which exhibit some selfsimilarity at very small times. Self-similar, singular-like structure has been observed in collapsing vortex rings just prior to core overlapping (Fernandez, Zabusky, \& Gryanik, 1995). More broadly, self-similar dynamics are useful in the study of vortex collapse and turbulence (Das, Kida, \& Goto, 2001; del Álamo et al., 2006; Kimura, 1987, 2009, 2010; Yoshimoto \& Goto, 2007).

Despite the interest in self-similar dynamics throughout the vortex dynamics and turbulence community, there has not been a systematic study concerning the existence of self-similar solutions to the full non-local Biot-Savart dynamics. In this paper, we demonstrate that not only are self-similar filament structures possible under the Biot-Savart dynamics (1.1), but also that they must be of the same power-law form found for the LIA. Employing a standard technique for regularizing the Biot-Savart integral near the core of the vortex filament, we may effectively partition solutions into an LIA part and a part which holds the contribution from the tails of the Biot-Savart integral. This allows us to construct asymptotic solutions and then compare such solutions to the existing LIA solutions. Indeed, we find that the self-similar solutions should be in strong qualitative agreement with those previously found for the LIA, meaning that the LIA is useful for approximating the self-similar filament solutions, even when they have kinks. The selfsimilar solutions obtained are for open filaments, which can be viewed as perturbations of line filaments into new configurations. We also show that closed filaments do not exhibit self-similarity for any power-law which preserved length, meaning that such filaments would monotonically increase or decrease in total arclength over time. These results lend credibility to the qualitative accuracy and robustness of the self-similar solutions previously known for the LIA.

\section{Self-similar vortex filament structure}

Consider a space curve $\mathbf{r}(s, t)$ where $s \in \mathbb{R}$ is the arclength variable while $t \geq 0$ is time. Then, $\mathbf{r}(s, t)$ gives a vortex filament solution provided that it satisfies the nonlocal dynamics given by equation (1.1). Since $\kappa>0$, we can scale the time variable like $t=\frac{2 \pi \hat{t}}{\kappa}$ (leaving only a factor of $1 / 2$ multiplying the integral in (1.1)). Let us consider a solution $\mathbf{r}(s, t)$ with the self-similar structure $\mathbf{r}(s, t)=\hat{t}^{a} \mathbf{R}(\eta)$ where $\eta=s \hat{t}^{b}$. While such solutions are known to exist for $a=1 / 2$ and $b=-1 / 2$ in the case of the LIA (Bewley et al., 2008; Lipniacki, 2000, 2003a,b; Van Gorder, 2013), it is not clear exactly what the scalings should be for the non-local equation (1.1). Placing this solution form into (1.1), 
we obtain

$$
\hat{t}^{a-1}\left(a \mathbf{R}(\eta)+b \eta \mathbf{R}^{\prime}(\eta)\right)=\frac{1}{2} \hat{t}^{-a} \int_{\hat{\ell}} \frac{(\mathbf{R}(\hat{\eta})-\mathbf{R}(\eta)) \times \mathbf{R}^{\prime}(\hat{\eta})}{|\mathbf{R}(\hat{\eta})-\mathbf{R}(\eta)|^{3}} d \hat{\eta},
$$

where prime denotes differentiation with respect to the similarity variable $\eta$ and $\hat{\ell}$ denotes the scaled vortex filament in the similarity frame of reference. Clearly, we must have $a=1 / 2$, which is the same as for the LIA. Unlike in the LIA, the scaling parameter $b$ is not specified from the equation. However, note that a line filament should be a solution to both the LIA and Biot-Savart dynamics. If we want a line in the self-similar coordinates to correspond with a line in the arclength coordinates, we should have $s=\hat{t}^{1 / 2} \eta=\hat{t}^{1 / 2+b} s$, meaning $b=-1 / 2$. Therefore, although the choice of $b=-1 / 2$ is not forced by the governing equation (as is the case for the LIA), we still find it convenient to make this selection so that line filaments in the self-similar frame map to line filaments in the arclength frame. With this, the governing equation in the self-similar frame becomes

$$
\mathbf{R}(\eta)-\eta \mathbf{R}^{\prime}(\eta)=\int_{\hat{\ell}} \frac{(\mathbf{R}(\hat{\eta})-\mathbf{R}(\eta)) \times \mathbf{R}^{\prime}(\hat{\eta})}{|\mathbf{R}(\hat{\eta})-\mathbf{R}(\eta)|^{3}} d \hat{\eta} .
$$

There are two types of vortex filament solutions one might expect from (2.2). The first of these, open vortex filaments, consist of a non-intersecting curve of infinite length in $\mathbb{R}^{3}$. In Section 3 we consider the asymptotics of such solutions under this self-similar formulation. We are able to show that the obtained solutions are in qualitative agreement with self-similar solutions previously obtained under the LIA model. The second type of solution would be closed vortex filaments, consisting of finite length closed curves in $\mathbb{R}^{3}$. Such solutions would model vortex rings, knotted vortex filaments, and so on. In Section 4 we consider the question of whether any such closed vortex filament solutions can exhibit self-similarity.

\section{Asymptotics for the open self-similar vortex filament}

Let us first consider an open vortex filament, which is a filament of infinite length that does not reconnect or self-intersect. Many vortex filaments studied in the literature, including helices, planar filaments, and self-similar filaments, fall into this category. For such a case, the arclength parameter takes all real values, meaning that the integration over $\hat{\ell}$ is equivalent to a parametric integration over the real line. Taking note of this, and then writing the vector $\mathbf{R}(\eta)=(x(\eta), y(\eta), z(\eta))$, we can write the coupled integrodifferential equations for each of the scalar components in (2.2) as

$$
\begin{aligned}
& x(\eta)-\eta x^{\prime}(\eta)=\int_{-\infty}^{\infty} \frac{(y(\hat{\eta})-y(\eta)) z^{\prime}(\hat{\eta})-(z(\hat{\eta})-z(\eta)) y^{\prime}(\hat{\eta})}{\left\{(x(\hat{\eta})-x(\eta))^{2}+(y(\hat{\eta})-y(\eta))^{2}+(z(\hat{\eta})-z(\eta))^{2}\right\}^{3 / 2}} d \hat{\eta}, \\
& y(\eta)-\eta y^{\prime}(\eta)=\int_{-\infty}^{\infty} \frac{(z(\hat{\eta})-z(\eta)) x^{\prime}(\hat{\eta})-(x(\hat{\eta})-x(\eta)) z^{\prime}(\hat{\eta})}{\left\{(x(\hat{\eta})-x(\eta))^{2}+(y(\hat{\eta})-y(\eta))^{2}+(z(\hat{\eta})-z(\eta))^{2}\right\}^{3 / 2}} d \hat{\eta}, \\
& z(\eta)-\eta z^{\prime}(\eta)=\int_{-\infty}^{\infty} \frac{(x(\hat{\eta})-x(\eta)) y^{\prime}(\hat{\eta})-(y(\hat{\eta})-y(\eta)) x^{\prime}(\hat{\eta})}{\left\{(x(\hat{\eta})-x(\eta))^{2}+(y(\hat{\eta})-y(\eta))^{2}+(z(\hat{\eta})-z(\eta))^{2}\right\}^{3 / 2}} d \hat{\eta} .
\end{aligned}
$$

From the system (3.1)-(3.3), it is clear to see that a line filament $\mathbf{R}(\eta)=\left(c_{1} \eta, c_{2} \eta, c_{3} \eta\right)$ is always a solution, and this immediately gives the line filament $\mathbf{r}(s, t)=\left(c_{1} s, c_{2} s, c_{3} s\right)$ in arclength coordinates. Many of the self-similar open filament solutions to the LIA in the literature are for Kelvin wave type structures along line filaments, and these can be realized as small perturbations of the line filament. One typically selects a single

coordinate along which to orient the filament curve, and without loss of generality (due 
to the symmetry of the equations (3.1)-(3.3)) we shall select the $x$ coordinate. Let us take $\epsilon>0$ to be a small amplitude parameter, which measures the small deviations of the waves along a line filament. Consider then a solution of the form

$$
x(\eta)=\eta+\epsilon^{2} X(\eta)+O\left(\epsilon^{4}\right), \quad y(\eta)=\epsilon Y(\eta)+O\left(\epsilon^{3}\right), \quad z(\eta)=\epsilon Z(\eta)+O\left(\epsilon^{3}\right) .
$$

We shall consider solutions with $X, Y$, and $Z$ that are $O(1)$ in epsilon to ensure that the perturbations remain valid. Terms that are $O\left(\epsilon^{3}\right)$ and smaller shall be neglected, since it is physically reasonable to take the amplitude of the perturbations $0<\epsilon<<1$. Placing (3.4) into (3.1)-(3.3), we obtain

$$
\begin{aligned}
& X(\eta)-\eta X^{\prime}(\eta)=\int_{-\infty}^{\infty} \frac{(Y(\hat{\eta})-Y(\eta)) Z^{\prime}(\hat{\eta})-(Z(\hat{\eta})-Z(\eta)) Y^{\prime}(\hat{\eta})}{|\hat{\eta}-\eta|^{3}} d \hat{\eta}+O\left(\epsilon^{2}\right), \\
& Y(\eta)-\eta Y^{\prime}(\eta)=\int_{-\infty}^{\infty} \frac{(Z(\hat{\eta})-Z(\eta))-(\hat{\eta}-\eta) Z^{\prime}(\hat{\eta})}{|\hat{\eta}-\eta|^{3}} d \hat{\eta}+O\left(\epsilon^{2}\right) \\
& Z(\eta)-\eta Z^{\prime}(\eta)=\int_{-\infty}^{\infty} \frac{(\hat{\eta}-\eta) Y^{\prime}(\hat{\eta})-(Y(\hat{\eta})-Y(\eta))}{|\hat{\eta}-\eta|^{3}} d \hat{\eta}+O\left(\epsilon^{2}\right)
\end{aligned}
$$

Note that (3.6)-(3.7) decouple from the function $X(\eta)$. Meanwhile, equation (3.5) has an integral term involving $Y(\eta)$ and $Z(\eta)$ alone. Therefore, one may solve the two equations (3.6)-(3.7) for $Y(\eta)$ and $Z(\eta)$ and then substitute the solution into (3.5) to obtain $X(\eta)$.

In order to find the solution pair to (3.6)-(3.7), we shall exploit the symmetry of the equations. Multiplying (3.7) by $i(i=\sqrt{-1})$ and adding the two equations, we obtain an equation of the form

$$
\Phi-\eta \Phi^{\prime}=i \int_{-\infty}^{\infty} \frac{(\hat{\eta}-\eta) \Phi^{\prime}(\hat{\eta})-(\Phi(\hat{\eta})-\Phi(\eta))}{|\hat{\eta}-\eta|^{3}} d \hat{\eta}
$$

where $\Phi(\eta)=Y(\eta)+i Z(\eta)$ is a complex-valued function. Once $\Phi(\eta)$ is found, we can recover the desired quantities by noting $Y(\eta)=\operatorname{Re}[\Phi(\eta)]$ and $Z(\eta)=\operatorname{Im}[\Phi(\eta)]$.

While the integration in (3.8) is over the real line, in principle the vortex filament is a very thin hollow tube, so there is a small core radius around each point, $|\eta-\hat{\eta}|<\delta$ with $\delta>0$, within which the integral does not apply. We assume that $\delta<<\epsilon<<1$, which is consistent with waves along the filament having much larger amplitudes than the vortex core radius, as is common in experiments and indeed assuming in the framework of the model. Removing this small region from the integral, and merging the integrals 
over $-\infty<\hat{\eta}<-\delta$ and $\delta<\hat{\eta}<\infty$, we obtain

$$
\begin{aligned}
& \int_{-\infty}^{\infty} \frac{(\hat{\eta}-\eta) \Phi^{\prime}(\hat{\eta})-(\Phi(\hat{\eta})-\Phi(\eta))}{|\hat{\eta}-\eta|^{3}} d \hat{\eta} \\
&= \int_{-\infty}^{\infty} \frac{\chi \Phi^{\prime}(\eta+\chi)-(\Phi(\eta+\chi)-\Phi(\eta))}{|\chi|^{3}} d \hat{\eta} \\
&=-2 \ln (\delta) \Phi^{\prime \prime}(\eta)+\int_{\delta}^{\infty} \frac{\chi \Phi^{\prime}(\eta+\chi)-(\Phi(\eta+\chi)-\Phi(\eta))}{\chi^{3}} d \chi \\
& \quad+\int_{-\infty}^{-\delta} \frac{\chi \Phi^{\prime}(\eta+\chi)-(\Phi(\eta+\chi)-\Phi(\eta))}{-\chi^{3}} d \chi \\
&=-2 \ln (\delta) \Phi^{\prime \prime}(\eta)+\int_{\delta}^{\infty} \frac{\chi \Phi^{\prime}(\eta+\chi)-(\Phi(\eta+\chi)-\Phi(\eta))}{\chi^{3}} d \chi \\
& \quad+\int_{\delta}^{\infty} \frac{-\chi \Phi^{\prime}(\eta-\chi)-(\Phi(\eta-\chi)-\Phi(\eta))}{\chi^{3}} d \chi \\
&=-2 \ln (\delta) \Phi^{\prime \prime}(\eta)+\int_{\delta}^{\infty} \frac{\chi\left[\Phi^{\prime}(\eta+\chi)-\Phi^{\prime}(\eta-\chi)\right]-[\Phi(\eta+\chi)+\Phi(\eta-\chi)-2 \Phi(\eta)]}{\chi^{3}} d \chi
\end{aligned}
$$

which leaves us with the equation

$\Phi-\eta \Phi^{\prime}=-2 \ln (\delta) i \Phi^{\prime \prime}+i \int_{\delta}^{\infty} \frac{\chi\left[\Phi^{\prime}(\eta+\chi)-\Phi^{\prime}(\eta-\chi)\right]-[\Phi(\eta+\chi)+\Phi(\eta-\chi)-2 \Phi(\eta)]}{\chi^{3}} d \chi$.

In the core region, $|\eta-\hat{\eta}|<\delta$, we have regularized by using the LIA (neglecting $O\left(\epsilon^{2}\right)$ terms that would give $O\left(\epsilon^{3}\right)$ corrections). This regularization method has been suggested and used in numerical studies of superfluid vortex filament dynamics (Adachi, Fujiyama, \& Tsubota 2010; Schwarz, 1985), and the appeal of this method is that it permits one to directly compare the LIA solutions with the Biot-Savart solutions. This regularization was recently employed to study helical filaments under (1.1) by Van Gorder (2015b), where the results were used to directly compare the Biot-Savart and LIA solutions. It was also used to regularize the planar filaments of Van Gorder (2015c).

Let us make an observation. In the case where the integral is approximated by LIA, the integral term goes like $\Phi^{\prime \prime}$ which gives an equation $\Phi-\eta \Phi^{\prime} \sim-i \Phi^{\prime \prime}$. This equation has solutions of the form $\Phi(\eta) \sim c_{1} \eta+c_{2} \eta \phi(\eta)$, where $\phi(\eta)$ satisfies $-\eta^{2} \phi^{\prime}=-i\left(2 \phi^{\prime}+\right.$ $\left.\eta \phi^{\prime \prime}\right)$. This implies $\phi^{\prime}(\eta)$ is of the form $\phi^{\prime}(\eta) \sim \eta^{-2} \exp \left(-\frac{i}{2} \eta^{2}\right)$. Separating into real and imaginary parts and integrating, one recovers $\Phi(\eta)$. Such a solution is possible as the small amplitude perturbations along the filament are effectively linear waves (plus negligibly small nonlinear contributions). Notice that while more complicated, equation (3.10) is linear in $\Phi(\eta)$. Therefore, a similar approach may work in the present case, as well. Since we are concerned with small perturbations along a filament which is directed along the $x(\eta)$ component, and since amplitude scalings at this order are negligible (since (3.10) is linear in $\Phi(\eta))$, we will assume a solution of the form

$$
\Phi(\eta)=\eta \phi(\eta) \quad \text { where } \quad \phi^{\prime}(\eta)=\frac{1}{\eta^{2}} \exp \left(i \rho \eta^{2}\right)
$$

where $\rho$ is a parameter to be determined. Note that the other linearly independent solution, $\Phi(\eta)=\eta$, is neglected, as it would just result in a $O(\epsilon)$ pivot in the $O(1)$ line filament. We would then simply reorient the coordinate frame to remove this pivot. Placing (3.11) into (3.10), we obtain the algebraic relation

$$
-1=-2 \rho \ln (\delta)+\rho(1-\gamma-2 \ln (\delta))+O\left(\delta^{2}\right) .
$$


The first term on the right hand side is the LIA contribution from the core, while the second term is the non-local contribution from the outer region. The parameter $\gamma$ is the Euler-Mascheroni constant $(\gamma \approx 0.5772)$. Since $\delta<<1$, we neglect $O\left(\delta^{2}\right)$ contributions from the integral. We therefore find

$$
\rho=\frac{-1}{1-\gamma-4 \ln (\delta)}
$$

Compare this to the pure LIA result, which would give $\rho=\frac{-1}{2|\ln (\delta)|}$, about double the value of (3.13), where non-local effects are included. Hence, non-locality is expected to modify the spatial scaling of these solutions, while the general qualitative properties should be consistent between the LIA and the Biot-Savart formulations.

Since $\delta<<1, \ln (\delta)<0$ and $\rho<0$, and hence the expression (3.13) gives $\Phi(\eta)=$ $\eta \int_{0}^{\eta} \sigma^{-2} \exp \left(-i|\rho| \sigma^{2}\right) d \sigma$. One can separate this into real and imaginary parts and perform the integration, recovering $Y(\eta)=\operatorname{Re}[\Phi(\eta)]=-\cos \left(|\rho| \eta^{2}\right)-\sqrt{2 \pi|\rho|} \eta S(\sqrt{2|\rho| / \pi} \eta)$ and $Z(\eta)=\operatorname{Im}[\Phi(\eta)]=\sin \left(|\rho| \eta^{2}\right)-\sqrt{2 \pi|\rho|} \eta C(\sqrt{2|\rho| / \pi} \eta)$. The functions $S$ and $C$ denote the Fresnel $\mathrm{S}$ and $\mathrm{C}$ functions, respectively.

From regularization of the integral in (3.5), and using our complex function $\Phi(\eta)$ as found above, we can write the integral as a function of $\eta$, obtaining

$$
M(\eta ; \delta)=\frac{1}{2 i} \int_{|\hat{\eta}-\eta|>\delta} \frac{\Phi^{*}(\hat{\eta}) \Phi^{\prime}(\hat{\eta})-\Phi(\hat{\eta}) \Phi^{*}(\hat{\eta})^{\prime}+\Phi(\eta) \Phi^{*}(\hat{\eta})^{\prime}-\Phi^{*}(\eta) \Phi^{\prime}(\hat{\eta})}{|\hat{\eta}-\eta|^{3}} d \hat{\eta} .
$$

From the form of $M$ defined in (3.14), we have $M(\eta ; \delta)=\epsilon^{2} \tilde{M}(\eta ; \delta)$ for $\tilde{M}=O(1)$ in $\epsilon$. Note that the integral in (3.14) is purely imaginary, therefore $\tilde{M}(\eta ; \delta)$ is a real-valued function. In order to solve for $X(\eta)$ from (3.5), note that (3.5) is equivalent to

$$
-\eta^{2} \frac{d}{d \eta}\left(\eta^{-1} X(\eta)\right)=\epsilon^{2} \tilde{M}(\eta ; \delta)+O\left(\epsilon^{3}\right),
$$

and manipulating and then integrating (3.15) we obtain

$$
\eta^{-1} X(\eta)=X_{0}-\epsilon^{2} \int_{\eta_{0}}^{\eta} \sigma^{-2} \tilde{M}(\sigma ; \delta) d \sigma+O\left(\epsilon^{3}\right)
$$

where $X_{0}>0$ is an arbitrary constant of integration and $\eta_{0}$ is some arbitrary value of $\eta$ corresponding to the lower bound of integration. Isolating (3.16) for $X(\eta)$, we have

$$
X(\eta)=X_{0} \eta-\epsilon^{2} \eta \int_{\eta_{0}}^{\eta} \sigma^{-2} M(\sigma ; \delta) d \sigma+O\left(\epsilon^{3}\right)
$$

With this, we have obtained the needed functions to describe the self-similar propagation of waves along a vortex filament up to order $O\left(\epsilon^{2}\right)$ contributions, where $\epsilon$ is the amplitude of the small wave-like perturbations. Mapping the above analysis into the original coordinate frame, the solution which consists of small amplitude Kelvin waves along a vortex filament is given by

$$
\mathbf{r}(s, t)=\left[\begin{array}{c}
\left\{X_{0}-\epsilon^{2} \int_{\eta_{0}}^{\sqrt{\frac{2 \pi}{\kappa t}} s} \sigma^{-2} \tilde{M}(\sigma ; \delta) d \sigma\right\} s \\
\left.-\epsilon\left\{\sqrt{\frac{\kappa t}{2 \pi}} \cos \left(\frac{2 \pi s^{2}}{(1-\gamma-4 \ln (\delta)) \kappa t}\right)+s \sqrt{\frac{2 \pi}{1-\gamma-4 \ln (\delta)}} S\left(\frac{2 s}{\sqrt{(1-\gamma-4 \ln (\delta)) \kappa t}}\right)\right\}\right]+O\left(\epsilon^{3}\right) . \\
\epsilon\left\{\sqrt{\frac{\kappa t}{2 \pi}} \sin \left(\frac{2 \pi s^{2}}{(1-\gamma-4 \ln (\delta)) \kappa t}\right)-s \sqrt{\frac{2 \pi}{1-\gamma-4 \ln (\delta)}} C\left(\frac{2 s}{\sqrt{(1-\gamma-4 \ln (\delta)) \kappa t}}\right)\right\}
\end{array}\right]
$$

This solution suggests several interesting things. First, self-similar solutions are indeed 
possible under the non-local Biot-Savart dynamics, and they have been shown here assuming small perturbations of a line filament. The dynamics are accurate to $O\left(\epsilon^{2}\right)$ in the small amplitude $0<\epsilon<<1$. Second, the structure of such self-similar solutions is essentially the same as seen under the LIA. Indeed, for small amplitude perturbations, inclusion of the non-local term (instead of approximating it with LIA) gives a spatial scaling of the aforementioned LIA similarity solutions. That is, $\mathbf{r}(s, t)=\mathbf{r}_{\mathrm{LIA}}(\tilde{s}, \tilde{t})$, where $\mathbf{r}_{\text {LIA }}$ is the corresponding solution under LIA, $\tilde{s}$ is a scaling of the arclength variable, $s$, and $\tilde{t}$ is a scaling of time, $t$. Third, while it has been known for some time that the helical filament exists under both the non-local Biot-Savart formulation and the LIA (see Van Gorder (2015b) and references therein), and recently it was shown that planar filaments exist under the Biot-Savart formulation (Van Gorder, 2015c), it has never been shown that similarity solutions exist under the Biot-Savart formulation. Unlike in the helical case, where tightly coiled helix solutions exist under the Biot-Savart formulation yet are not accurately reproduced under the LIA, here the self-similar vortex filament structure is qualitatively similar under both Biot-Savart and LIA formulations. This is a feature which is shared with the planar vortex filament, which qualitatively is the same under both non-local and local formulations (with only minor differences in scaling of the space and temporal variables).

Putting (3.18) into Cartesian coordinates instead of arclength, we have that the filament position curve can we written in terms of the single spatial variable, $x$, and time, $t$, like

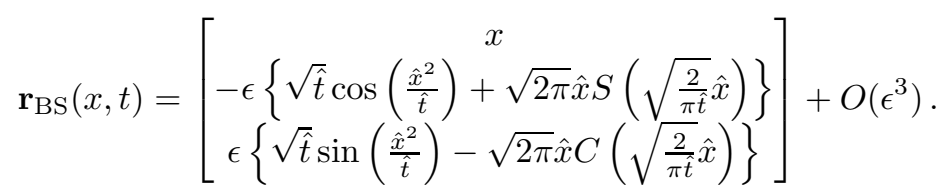

In the $y$ and $z$ components, we have used the scalings

$$
\hat{t}=\frac{\kappa}{2 \pi} t \quad \text { and } \quad \hat{x}=\frac{1}{\sqrt{1-\gamma-4 \ln (\delta)}} \frac{x}{X_{0}}
$$

in order to simplify the representations. Observe that, if we had instead neglected integral terms and kept only the inner terms, we would have recovered the LIA solution, which in our notation would be given by

$$
\mathbf{r}_{\mathrm{LIA}}(x, t)=\left[\begin{array}{c}
x \\
-\epsilon\left\{\sqrt{\hat{t}} \cos \left(\frac{\mu^{2} \hat{x}^{2}}{\hat{t}}\right)+\sqrt{2 \pi} \mu \hat{x} S\left(\sqrt{\frac{2}{\pi t}} \mu \hat{x}\right)\right\} \\
\epsilon\left\{\sqrt{\hat{t}} \sin \left(\frac{\mu^{2} \hat{x}^{2}}{\hat{t}}\right)-\sqrt{2 \pi} \mu \hat{x} C\left(\sqrt{\frac{2}{\pi t}} \mu \hat{x}\right)\right\}
\end{array}\right]+O\left(\epsilon^{3}\right) .
$$

Note the appearance of a scaling parameter, $\mu=\mu(\delta)$, which is defined by

$$
\mu(\delta)=\sqrt{\frac{1-\gamma-4 \ln (\delta)}{-2 \ln (\delta)}}=\sqrt{2+\frac{1-\gamma}{2 \ln \left(\delta^{-1}\right)}}>\sqrt{2},
$$

for $0<\delta<<1$. Variations in the LIA filament curve are therefore more tightly centered around the origin, as the $y$ and $z$ components of the filament, which give the waves, scale as a dilation of the Biot-Savart solution. In particular, $y_{\mathrm{LIA}}(x, t)=y_{\mathrm{BS}}(\mu x, t)$ and $z_{\mathrm{LIA}}(x, t)=z_{\mathrm{BS}}(\mu x, t)$ for $\mu>\sqrt{2}$. Since the LIA dynamics are scaled toward the origin, for any fixed behavior of the Biot-Savart solution on a spatial interval $[-L, L]$, these same dynamics are contained in an open spatial interval $(-L / \sqrt{2}, L / \sqrt{2})$ when we go to the LIA solutions. Therefore, the Biot-Savart dynamics are more smooth than the 


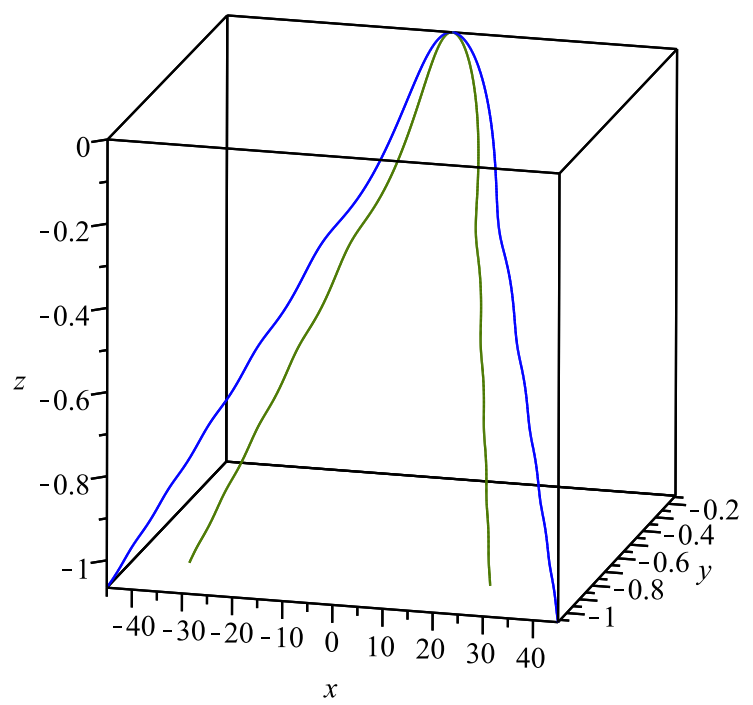

FiguRE 1. Comparison of the regularized Biot-Savart (blue curve) and LIA (green curve) vortex filament solutions given by (3.19) and (3.21), respectively. Parameter values are $\epsilon=0.1$, $\delta=0.001$, while the plots are given at time $\hat{t}=2$. While qualitatively similar, the LIA filament has a sharper kink at the origin. As suggested by the scaling ratio satisfying $\mu>2$, we have that the LIA solutions will have sharper kinks at the origin than the corresponding Biot-Savart solutions.

LIA dynamics. This makes sense, as the integral in the Biot-Savart dynamics results in a smoothing of solutions when compared to the LIA (which only involves differentiation).

In Figure 1, we compare the Biot-Savart solutions (3.19) with the LIA solutions (3.21). Any kinks that form at the center will necessarily be more sharp under LIA than for the Biot-Savart formulation, due to the scaling property discussed above. In Figure 2, we observe the temporal dynamics of a self-similar vortex filament under the Biot-Savart law. As time progresses, the Kelvin waves radiate outward along the vortex filament, resulting in a smoothing of the structure. Conversely, one observes very sharp kinks in the singular limit $t \rightarrow 0^{+}$. These kinks are smoothed as the central region sheds Kelvin waves.

While the angle of the kinks located at the origin shown in Figures 1 and 2 appear rather obtuse, this is due to the choice of spatial scaling, since $X_{0}=1$ in those plots. If this scaling factor is decreased, the angle formed decreases, and vice versa. Meanwhile, if $\epsilon$ is increase, the angle formed decreases. While the analytical theory outlined here is valid for sufficiently small $\epsilon$, a similar trend should be observed in any numerical simulations (provided again that the scaling factor is chosen appropriately). The core parameter, $\delta$, is taken to be less than $\epsilon$ for physical reasons, although changes are apparent even for small changes in $\delta$. These results are demonstrated in Figure 3.

In order to analytically calculate the angles formed for very small $t$, as shown in Figure 3 , we took the $t \rightarrow 0$ limit of the approximate solution (3.19). This limit resulted in two rays emanating from the origin, one corresponding to $x>0$, the other corresponding to $x<0$. From standard vector calculus, the angle between these two (which corresponds to the angle of the kink seen in Figures 1 and 2 for very small time), is given by

$$
\theta_{\mathrm{BS}}=180^{\circ}-\arccos \left(\frac{1-\frac{\pi \epsilon^{2}}{2 X_{0}^{2}|\ln (\delta)|}}{1+\frac{\pi \epsilon^{2}}{2 X_{0}^{2}|\ln (\delta)|}}\right) \frac{180^{\circ}}{\pi},
$$




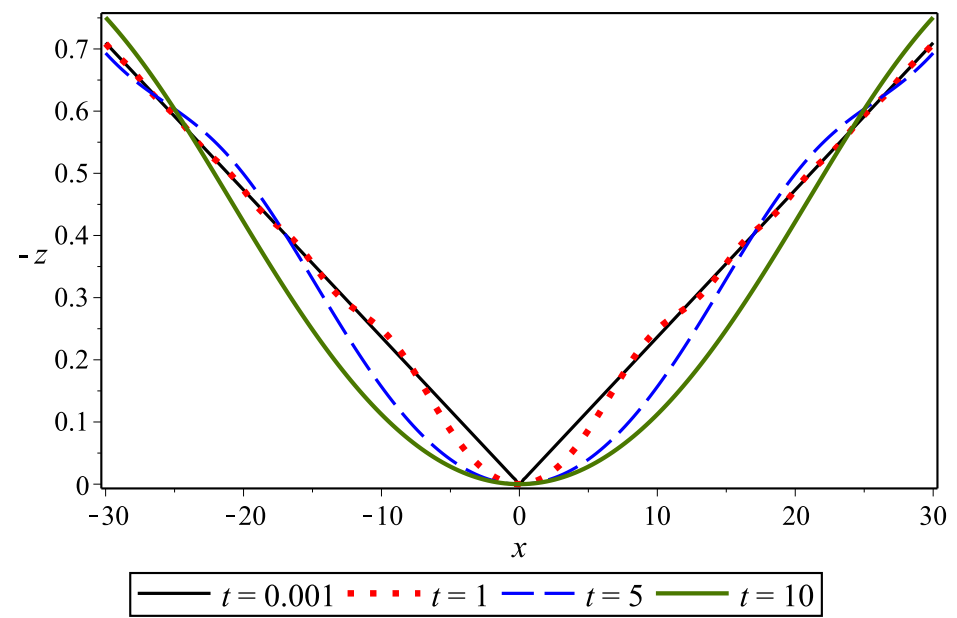

Figure 2. Time evolution of a regularized Biot-Savart vortex filament solutions given by (3.19). Parameter values are $\epsilon=0.1, \delta=0.001$, while we sample a variety of times. For ease of viewing, we plot solutions in the $(x,-z)$ plane. As time increases, the sharp kink at the origin is smoothed, while the filament radiates Kelvin waves radially outward from this kink. Note that we have picked the scaling parameter to be $X_{0}=1$. For other values, we may make the kink at the origin more or less sharp at $t=0$, with the structure being smoothed as $t$ increases.

in units of degrees. A corresponding result for the angle formed at the origin by the kink in the LIA solutions (3.21) is found to be

$$
\theta_{\text {LIA }}=180^{\circ}-\arccos \left(\frac{1-\frac{\pi \mu(\delta)^{2} \epsilon^{2}}{2 X_{0}^{2}|\ln (\delta)|}}{1+\frac{\pi \mu(\delta)^{2} \epsilon^{2}}{2 X_{0}^{2}|\ln (\delta)|}}\right) \frac{180^{\circ}}{\pi},
$$

We find that wider filaments (corresponding to larger values of $\delta$ ) result in sharper kinks, and hence appear to have less regularity. As the ratio $\epsilon / X_{0}$ increases, the kinks will become sharper. Thus, solutions with larger spatial deflections or more rapid spatial variation can have sharper kinks for small time. Note that the results plotted in Figure 3 correspond to the $t \rightarrow 0$ limit. When $t$ increases, the kink initially at the origin will smooth in all cases, as the kink radiates Kelvin waves; these dynamics were seen in Figure 2 for the Biot-Savart case, and hold true for other parameter values (as well as for the corresponding LIA solutions). When comparing the Biot-Savart solutions with the LIA solutions, note that the LIA solutions result in sharper initial kinks given the same parameter values. This is in agreement with the view that the Biot-Savart dynamics smooth the filament structures. Still, the dynamics emergent for each of the Biot-Savart and LIA models are in complete qualitative agreement, suggesting that the self-similar structures observed in the literature under the LIA are likely valid under more complicated (and more computationally involved) Biot-Savart dynamics.

\section{On the existence of a self-similar closed vortex filament}

A closed vortex filament is a vortex filament of finite length which closes on itself (one may connect one end of an open filament of finite length to the other in order to close the filament curve). The primary example of such a filament is a curve corresponding to a thin vortex ring. The parameterization with arclength can therefore be viewed as periodic, say $0<s<2 \pi$, and this gives the bounds for the integration over $\ell$. Now, 


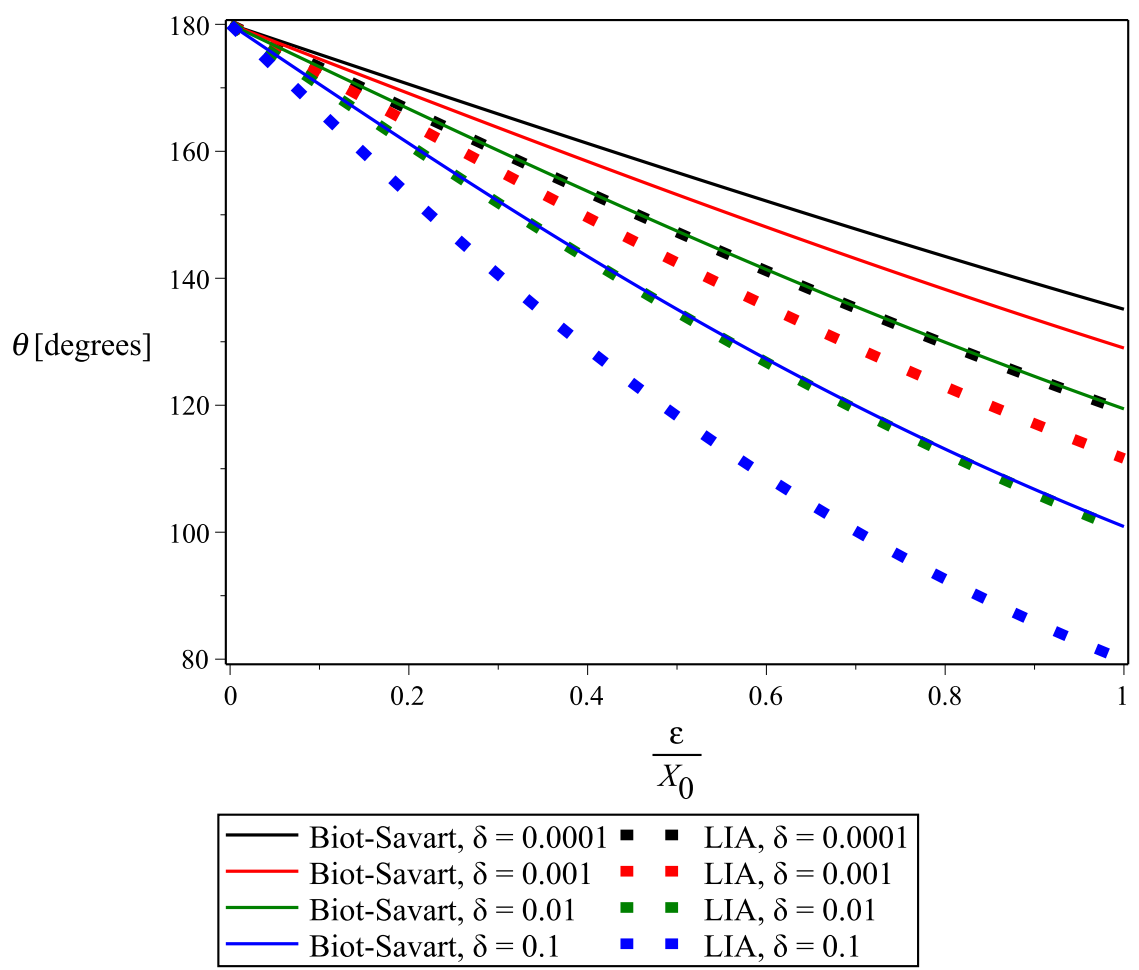

Figure 3. Comparison of the interior angle $\theta$ in the kink solutions near the origin for small time under both Biot-Savart (3.23) and LIA (3.24) models. We plot the angles over the ratio $\epsilon / X_{0}$, which is the ratio of the deflection extent to the spatial scaling, as this ratio is what enters into the analytical formulas (3.23) and (3.24). As the ratio increases (that is, as the spatial scaling becomes compatible with the deflection size), the kink in the filament at $t=0$ becomes more sharp. Furthermore, the role of the core radius parameter, $\delta$, is to make this kink more sharp. This means that the more thick the filament, the more obtuse the angle will tend to be. Note also that the LIA solutions result in uniformly more share kinks in all cases. This makes sense, in light of the fact that the non-local integral part of the Biot-Savart dynamics was suggested to have a smoothing effect on the solutions. However, these plots make it clear that the smoothing effects do not modify the qualitative features of self-similar solutions previously observed under the LIA.

for the parameterization with $\eta$, since the arclength is bounded the end points are in general time dependent. Therefore, the integration over $\hat{\ell}$ will take place on the interval $0<\eta<\frac{2 \pi}{\sqrt{t}}$. This temporal upper bound is undesirable for multiple reasons. First, this explicit time dependence means that there is not true similarity in the solution. Second, as time increases, the size of the closed filament tends to zero, even if the size is static in the arclength frame. Finally, as time is taken to zero, the length in the new coordinate becomes infinite. For these reasons, the type of similarity transform used above is not particularly helpful for studying closed filaments.

We would like a solution which would maintain the length parameterization of the closed curve. Recall that we picked the similarity parameter $b=-1 / 2$ so that line filaments would be mapped to line filaments. As line filaments are never closed curves, we should not be concerned with this here. Therefore, it makes more sense to pick $b$ in order to maintain the structure of the closed curve solutions for all time. For this reason, we pick $b=0$. This gives $\eta=s$, and the solution structure is $\mathbf{r}(s, t)=\sqrt{\hat{t}} \mathbf{R}(\eta)=\sqrt{\hat{t}} \mathbf{S}(s)$, where $\mathbf{S}(s)$ is a curve depending on arclength alone. The spatial structure of the curve 
can be found from solving

$$
\mathbf{S}(s)=\int_{\ell} \frac{(\mathbf{S}(\hat{s})-\mathbf{S}(s)) \times \mathbf{S}^{\prime}(\hat{s})}{|\mathbf{S}(\hat{s})-\mathbf{S}(s)|^{3}} d \hat{s},
$$

where again $s \in \mathbb{R}$ denotes arclength. Yet, since $\mathbf{S}$ depends only on arclength, this means $\mathbf{r}(s, t)$ is a static curve in space which is scaled by time in a multiplicative manner. At time $t=0$, the curve will emanate from a point $\mathbf{r}(s, 0)=(0,0,0)$ and as time progresses one will have a curve of the form $\mathbf{r}(s, t)=(\sqrt{\hat{t}} x(s), \sqrt{\hat{t}} y(s), \sqrt{\hat{t}} z(s))$ which maintains form but undergoes dilation as it expands in space. As time becomes infinitely large, the curve will enclose an infinite region. This behavior is the opposite of the type of behavior seen when we instead had taken $b=-1 / 2$. Therefore, each respective scaling will give a closed filament of infinite length for large $(b=0)$ or small $(b=-1 / 2)$ time, with contraction (dissipation) of the closed filament in the respective opposite temporal limits. We find that the $b=0$ behavior is representative of $b \geq 0$, while the $b=-1 / 2$ behavior is representative of $b<0$, hence the two types of behaviors given above are the only possible behaviors for self-similar closed filaments. Interestingly, this means that behaviors observed near core overlapping events during the collapse of vortex rings by Fernandez, Zabusky, \& Gryanik (1995) could not be sustained, and hence should only be observed in the small time limit. Rather, such dynamics could be viewed as perturbations of some self-similar process, with the perturbations dominating away from the core overlapping if one were to consider the time reversal of the process. We should point out the the collapse of a vortex filament to a point singularity in a manner consistent with a self-similarity scaling $\left(t-t_{0}\right)^{1 / 2}$ was described in Siggia (1985). In that study, Siggia used a modified Biot-Savart formulation in order to numerically simulate results. Note that a time reversal would then allow one to generate a filament of infinite length from a point singularity.

Ricca, Samuels, \& Barenghi (1999) considered perturbations of closed circular vortex filaments in order to study vortex knots under the Biot-Savart dynamics. One may wonder if such solutions satisfy a similarity scaling. As we've shown here, one needs $b=0$ for a self-similar closed filament. However, if we assume such a solution, and then place it into (4.1), we find that no circular curve can be a solution (no matter the choice of orientation, or parameters used such as the radius). As the results of Ricca, Samuels, \& Barenghi (1999) were obtained from perturbations about circular curves, it is clear that such solutions would not likely satisfy (4.1) after translation or parameter scaling. Therefore, we would not expect closed curves corresponding to vortex circular rings, not more complicated knotted filaments, to exhibit self-similarity.

We should also note that more general planar solutions than even circular solutions will not exist for (4.1). To show this, note that a planar solution can be written with two components without loss of generality, as one may always rotate the coordinate frame. Taking $\mathbf{S}(s)=(A(s), B(s), 0)$ such that $A$ and $B$ are $2 \pi$-periodic, we find that (4.1) gives

$$
\left[\begin{array}{c}
A(s) \\
B(s) \\
0
\end{array}\right]=\left[\begin{array}{c}
0 \\
0 \\
\int_{0}^{2 \pi} \frac{(A(\hat{s})-A(s)) B^{\prime}(\hat{s})-(B(\hat{s})-B(s)) A^{\prime}(\hat{s})}{\left\{(A(\hat{s})-A(s))^{2}+(B(\hat{s})-B(s))^{2}\right\}^{3 / 2}} d \hat{s}
\end{array}\right] .
$$

Therefore, for the planar case, the left and right hand sides of (4.1) are orthogonal to one another, and the only solution is the trivial solution $A=0$ and $B=0$. Hence, no planar solution exists.

In order to get a better feeling for whether a solution to (4.1) may actually exist, let us close this section by noting that for many filaments, there is some parameter limit 
where Biot-Savart dynamics agree at least qualitatively with those found under the LIA. We demonstrated this in the previous section for open filaments, and this is true of other types of solutions, such as helical filaments (when the filament is not tightly coiled; see Van Gorder (2015b)) and planar filaments (Van Gorder, 2015c). Therefore, to get an understanding of the qualitative features of a solution to (4.1), let us consider a relevant LIA approximation. Assuming the spatial variable $s$ is scaled appropriately, we may ignore the factor arising under LIA, and simply approximate (4.1) by

$$
\mathbf{S}=\mathbf{S}^{\prime} \times \mathbf{S}^{\prime \prime},
$$

where prime denotes differentiation with respect to $s$. Differentiation with respect to $s$ yields

$$
\mathbf{S}^{\prime}=\mathbf{S}^{\prime} \times \mathbf{S}^{\prime \prime \prime},
$$

which implies that either the right hand side is orthogonal to the left hand side (which makes no sense) or that $\mathbf{S}^{\prime}=\mathbf{0}$. Since only the latter case is possible, we must have that $\mathbf{S}(s)=\mathbf{S}^{0}$, a constant vector. Yet, the only constant solution to (4.3) is the zero solution. We have therefore shown that there exist no non-trivial solutions to (4.3). Then, in the case where the LIA and Biot-Savart solutions agree, there would exist no non-trivial solution to (4.1), meaning there would exist no closed self-similar vortex filament. Now, it may be that there could arise some pathological case which permits a non-trivial solution of (4.1) which does not correspond to a solution of (4.3). Hence, we suggest only that it is unlikely that a non-trivial solution to (4.1) exists, and we have no proof for the general Biot-Savart dynamics. It is therefore an open question whether a bounded and periodic solution actually exists for (4.1). Even if such a solution were to exist, it would maintain it's form while growing like $\sqrt{t}$ as $t$ increases. Such a solution may not be physical.

\section{Discussion}

We have shown that self-similar vortex filament solutions are possible under the nonlocal Biot-Savart model, and that such solutions are in qualitative agreement with LIA solutions studied previously (although we recreate such self-similar solutions here so that we may compare the two in the exact same parameter space and coordinate frame). This agreement is rather interesting, as it is certainly not universal among all solutions to these models. For instance, the helical vortex filament behaves rather differently between the Biot-Savart and LIA models in the case of large wave numbers (corresponding to a tightly coiled filament); see, for instance, Van Gorder (2015b) and references therein. Therefore, the self-similar case is one in which we can be confident that the Biot-Savart and LIA dynamics are in (qualitatively) good agreement for the dynamics of thin vortex filaments of sufficiently bounded deflection.

The primary difference in small-amplitude solutions under each of the Biot-Savart and LIA models is that, owing to the non-locality, the Biot-Savart model solutions exhibit a greater degree of smoothness, as evidenced by the fact that kinks arising for small times are sharper for the LIA solutions than for the Biot-Savart solutions. This further supports the idea that self-similar structures found for the LIA are physically reasonable vortex filament solutions (Van Gorder, 2015d). Physically, the vortex kink solutions arising from a similarity assumption correspond to the vortex filament structures previously studied experimentally in Fonda et al. $(2012,2014)$ following reconnection events. These reconnection events were considered theoretically in Kursa, Bajer,\& Lipniacki (2011), where it was shown that the post-intersection dynamics can involve the creation of vortex rings for small time, before the two predominant $\mathrm{V}$-shaped filaments dominate. At 
small times, the V-shaped filaments exhibit the largest amplitude Kelvin waves, while as time progresses, there is a smoothing effect and the waves along the filament gradually dissipate leaving a more smooth U-structure, and this is exactly the behavior we observe in Figure 2.

While most of this study was devoted to open vortex filaments, we deduced the necessary similarity form for a closed vortex filament, and it was shown to differ from that of the open filament. However, the standard closed filaments do not appear to exist under the similarity transform, which is equivalent to the statement that those known solutions (such as vortex rings or knots) do not exhibit self-similarity. Still, it may be possible to construct solutions to the integral equation (4.1), which would then result in closed filament solutions. This is therefore an open question.

It is worth mentioning that there is a quantum form of the LIA (Schwarz, 1985), which is essentially the LIA applied to the HVBK model (Hall \& Vinen, 1956a,b; Bekarevich \& Khalatnikov, 1961). It should be feasible to extend the present analytical results to that model, similar to what was done for the study of non-local dynamics of Kelvin waves under the Schwarz model (Van Gorder, 2015a). However, since the Biot-Savart and LIA results are shown to be in very good qualitative agreement here, we are comfortable that the existing LIA results for the Schwarz model are descriptive of the real physics, and that the addition of non-local terms would be more useful for computation or quantitative accuracy, particularly when considering mutual friction and normal fluid effects. It is worth noting that the non-local Biot-Savart description would also be useful for studying the interaction of multiple vortex filaments with self-similar structures.

\section{REFERENCES}

Adachi, H., Fujiyama, S., \& Tsubota, M. 2010 Steady-state counterflow quantum turbulence: Simulation of vortex filaments using the full Biot-Savart law. Phys. Rev. B 81(10), 104511.

Arms, R. J. \& Hama, F. R. 1965 Localized-induction concept on a curved vortex and motion of an elliptic vortex ring. Phys. Fluids 8, 553.

Bekarevich, I. L. \& Khalatnikov, I. M. 1961 Phenomenological derivation of the equations of vortex motion in He II. Sov. Phys. JETP 13, 643.

Bewley, G. P., Paoletti, M. S., Sreenivasan, K. R., \& Lathrop, D. P. 2008 Characterization of reconnecting vortices in superfluid helium. Proceedings of the National Academy of Sciences 105(37), 13707-13710.

Boersma, J. \& Wood, D. H. 1999 On the self-induced motion of a helical vortex. J. Fluid Mech. 384, 263.

Boffetta, G., Celani, A., Dezzani, D., Laurie, J., \& Nazarenko, S. 2009 Modeling Kelvin Wave Cascades in Superfluid Helium. J. Low Temp. Phys. 156, 193.

Da Rios, L. S. 1906 Sul moto d'un liquido indefinite con un filetto vorticoso di forma qualunque. Rend. Circ. Mat. Palermo 22, 117.

Das, C., Kida, S., \& Goto, S. 2001 Overall self-similar decay of two-dimensional turbulence. Journal of the Physical Society of Japan 70(4), 966-976.

del Álamo, J., Jimenez, J., Zandonade, P., \& Moser, R. D. 2006 Self-similar vortex clusters in the turbulent logarithmic region. J. Fluid Mech. 561, 329-358.

Fernandez, V. M., Zabusky, N. J., \& Gryanik, V. M. 1995 Vortex intensification and collapse of the Lissajous-elliptic ring: single-and multi-filament Biot-Savart simulations and visiometrics. J. Fluid Mech. 299, 289-331.

Fonda, E., Meichle, D.P., Ouellette, N.T., Hormoz, S., \& Lathrop, D.P. 2014 Direct observation of Kelvin waves excited by quantized vortex reconnection. Proc. Natl. Acad. Sci. USA 111, 4707.

Fonda, E., Meichle, D.P., Ouellette, N.T., Hormoz, S., Sreenivasan, K.R., \& Lathrop, D.P. 2012 Visualization of Kelvin waves on quantum vortices. arXiv:1210.5194.

Gutiérrez, S., Rivas, J., \& Vega, L. 2003 Formation of Singularities and Self-Similar Vortex Mo- 
tion Under the Localized Induction Approximation. Communications In Partial Differential Equations 28, 927-968.

Hall, H. E. \& Vinen, W. F. 1956a The rotation of liquid helium II. I. Experiments on the propagation of second sound in uniformly rotating helium II. Proc. R. Soc. Lond. A 238, 204.

Hall, H. E. \& Vinen, W. F. 1956b The rotation of liquid helium II. II. The theory of mutual friction in uniformly rotating helium II. Proc. R. Soc. Lond. A 238, 215.

Kimura, Y. 1987 Similarity solutions of two-dimensional point vortices. J. Phys. Soc. Jpn. 56, 2024-2030.

Kimura, Y. 2009 Self-similar collapse of a 3D straight vortex filament model. Geophys Astrophys Fluid. Dyn. 103, 135-142.

Kimura, Y. 2010 Self-similar collapse of 2D and 3D vortex filament models. Theoretical and Computational Fluid Dynamics, 24(1-4), 389-394.

Kursa, M., Bajer, K., \& Lipniacki, T. 2011 Cascade of vortex loops initiated by a single reconnection of quantum vortices. Phys. Rev. B 83(1), 014515.

Lipniacki, T. 2000 Evolution of quantum vortices following reconnection. European Journal of Mechanics-B/Fluids 19(3), 361-378.

Lipniacki, T. 2003a Quasi-static solutions for quantum vortex motion under the localized induction approximation. J. Fluid Mech. 477, 321-337.

Lipniacki, T. 2003b Shape-preserving solutions for quantum vortex motion under localized induction approximation. Phys. Fluids 15(6), 1381-1395.

Moin, P., Leonard, A., \& Kim, J. 1986 Evolution of a curved vortex filament into a vortex ring. Phys. Fluids 29(4), 955-963.

Pelz, R. B. 1997 Locally self-similar, finite-time collapse in a high-symmetry vortex filament model. Phys. Rev. E 55(2), 1617.

Ricca, R. L. 1991 Rediscovery of Da Rios equations. Nature 352, 561.

Ricca, R. L. 1994 The effect of torsion on the motion of a helical vortex filament. J. Fluid Mech. 273241.

Ricca, R. L., Samuels, D. C., \& Barenghi, C. F. 1999 Evolution of vortex knots, J. Fluid Mech. 391, 29-44.

Schwarz, K. W. 1985 Three-dimensional vortex dynamics in superfluid ${ }^{4}$ He: Line-line and lineboundary interactions. Phys. Rev. B 31, 5782.

Siggia, E. D. 1985 Collapse and amplification of a vortex filament. Phys. Fluids 28(3), 794-805.

Van Gorder, R. A. 2013 Self-similar vortex dynamics in superfluid 4He under the Cartesian representation of the Hall-Vinen model including superfluid friction. Phys. Fluids 25(9), 095105.

Van Gorder, R. A. 2014 Decay of helical Kelvin waves on a quantum vortex filament. Phys. Fluids 26, 075101.

Van Gorder, R. A. 2015a The Biot-Savart description of Kelvin waves on a quantum vortex filament in the presence of mutual friction and a driving fluid. Proc. R. Soc. A 471, 20150149.

Van Gorder, R. A. 2015b Helical vortex filament motion under the non-local Biot-Savart model. J. Fluid Mech. 762, 141-155.

Van Gorder, R. A. 2015c Non-local dynamics governing the self-induced motion of a planar vortex filament. Phys. Fluids 27, 065105.

Van Gorder, R. A. 2015d Translation of waves along quantum vortex filaments in the lowtemperature two-dimensional local induction approximation. Phys. Fluids 27, 095104.

Widnall, S. E. 1972 The stability of a helical vortex filament. J. Fluid Mech. 54, 641.

Yoshimoto, H., \& Goto, S. 2007 Self-similar clustering of inertial particles in homogeneous turbulence. J. Fluid Mech. 577, 275-286. 